\title{
Detection of Cryptococcus Neoformans Amongst HIV Patients Attending Comprehensive Care ClinicAt Kapkatet District Hospital, KENYA
}

\author{
R.K.A. Sang ${ }^{1}$, Sarah C. Maritim ${ }^{2}$, Kimathi Kobia ${ }^{3}$. \\ ${ }^{1}$ Egerton University (Faculty of Health Sciences), ${ }^{2}$ Kapkatet District Hospital (Medical Laboratory Services) \\ ${ }^{3}$ Mount Kenya University (School of Health Sciences)
}

\begin{abstract}
Cryptococcal meningitis is the most common lethal opportunistic fungal infection caused by a fungus (yeast) - Cryptococcus neoformans in people with HIV and AIDS. Cryptococcal infection affects approximately one million HIV-infected patients worldwide each year, and is associated with high mortality. In Sub-Saharan Africa, Cryptococcal disease has been associated with 17\% of all deaths among HIV-infected patients and 75\% of deaths from opportunistic infections in men with pulmonary tuberculosis. Prompt treatment depends on awareness of the common symptoms. In Sub-Saharan Africa, Kenya included, data on the prevalence of Cryptococcal infection is scanty. This cross-sectional study carried out at Kapkatet District Hospital Comprehensive Care Clinic (CCC) using 300 selected HIV patients enrolled for review at the CCC from June to August 2012 sought to identify HIV infected patients who also had Cryptococcus neoformans as detected from their blood samples. Serum samples were studied for the detection of capsular polysaccharide antigen of Cryptococcus neoformans usinglatex agglutination technique with pronase pre-treatment (IMMY, Crypto-Latex Antigen Detection System, Immunomycologics Inc., OK, USA). Results showed that $1 \%$ of the patients were confirmed to have Cryptococcus Neoformans. Of those found positive, $25 \%$ were males and $75 \%$ were females and were distributed between ages 21 and 50 years which also showed that $73 \%$ of all patients who participated in the study were in this age range. Since Cryptococcosis is highly lethal once contracted, health care workers managing people living with HIV/AIDS need to have ahigh index of suspicion so as to initiate prompt treatment immediately the disease is confirmed.
\end{abstract}

Abbreviations And Acronyms

AIDS------Acquired Immunodeficiency Syndrome

ART------Antiretroviral Treatment

CCC------Comprehensive Care Clinic

CD4------ It is a white blood cell that the HIV virus attacks.

CSF-------Cerebro Spinal Fluid

HAART -- Highly Active Antiretroviral Therapy

HIV-------Human Immunodeficiency Virus

PCR ------ Polymerase Chain Reaction

WHO-----World Health Organization

Operational Definitions

Morbidity----The burden of the disease.

Mortality-----The death rate.

Pathogen---- The disease causing organism.

Potency------The strength of a drug.

Sampling---- The process of selecting individuals or units for the sub group.

\section{1:Background Information}

\section{Introduction}

Cryptococcosis is caused by Cryptococcus neoformans, a thin-walled non-mycelial budding yeast (fungus) that is characterized by a thick polysaccharide capsule best seen on Indian ink stain (Parker et. al., 2009). As one of the most common opportunistic infections, Cryptococcal infection affects approximately one million HIV-infected patients worldwide each year, and is associated with high mortality (Parker et. al., 2009; Powderly et. al., 1995). In Sub-Saharan Africa, Cryptococcal disease has been associated with $17 \%$ of all deaths among HIV-infected patients (French et. al, 2002) and 75\% of deaths from opportunistic infections in men with pulmonary tuberculosis (Church et. al., 2000).Most HIV-infected individuals with cryptococcosis present with meningitis and, less commonly, with meningitis and pneumonia or isolated pneumonia.

Cryptococcus neoformans is the most common life threatening fungal pathogen in patients with acquired immuno-deficiency syndrome (AIDS) (Martha, et. al., 1996). It is the common cause of life threatening 
meningitis in AIDS with 5-8\% of patients with AIDS developing Cryptococcal infection. With effective antiretroviral treatment (ART), the prevalence of Cryptococcus along with other opportunistic infections has decreased (Michael, et.al.,1999). In particular, there is little data from Sub-Saharan Africa, Kenya included, on the prevalence of disorders associated with Cryptococcus neoformans.

Although the overall incidence of cryptococcosis is unknown, it is higher among patients with AIDS in Africa and South-east Asia than in the United States, whereas it appears less frequently in Europe (McGuire D, et al 1997). In the developed world, the introduction of potent antiretroviral therapies resulted in a decrease in the incidence of opportunistic infections associated with AIDS.

More than three fourths of the cases associated with AIDS develop when the CD4 T-lymphocyte count falls below 50 cells $/ \mu \mathrm{L}$. In a 1996 retrospective review of 65 AIDS patients with Cryptococcal meningitis in France, the median CD4 count was 46 cells/ $\mu \mathrm{L}$ (Pinner RW et al 1995). Cryptococcosis was the initial AIDS-defining illness in $63 \%$ of patients (Darras - Joly C et al, 1996).

\subsection{Statement of the problem}

The mortality rate of patients with Cryptococcal meningitis having AIDS is 10-25\%. In patients with AIDS, Cryptococcal meningitis is usually incurable, and individuals who survive the initial infection are given lifelong antifungal therapy to reduce the likelihood of relapse. Even when the infection has been treated, individuals may be left with a variety of complications such as, weakness, headache, paralysis, hearing and visual loss. As Cryptococcal meningitis occurs at a relatively advanced stage of AIDS, the affected people have reduced productivity and time lost from work may be significant. Because the symptoms are frequently nonspecific, and the symptoms of classic meningitis, such as stiff neck and aversion to light do not occur in many patients with Cryptococcal meningitis, awareness of meningitis is often missed by the person infected and consequently there is often a delay of several weeks or months before medical attention is sought.

\section{1: Broad objective}

\section{Study Objectives}

To determine the extent of cryptococcal infection amongst HIV/AIDS patients attending CCC at Kapkatet District Hospital.

\section{2: Specific objectives}

1. To determine the proportion of patients with cryptococcal infection among HIV/AIDS patients enrolled in CCC register who came for review at Kapkatet District Hospital.

2. To determine the proportion of patients with cryptococcal infection according to age and gender.

3. To determine the proportion of asymptomatic patients presenting with less than $300 \mathrm{CD} 4+$ cells/micro liters of blood who have cryptococcal infection.

\section{3: Research Question}

1. Is it always true that HIV clients can easily get cryptococcal infection?

\section{4: Hypothesis}

It is generally accepted that most patients who are immuno-suppressed and have HIV tend to have Cryptococcus neoformans infection.

\section{1: History of Cryptococcosis}

\section{Literature Review}

Although the overall incidence of cryptococcosis is unknown, it is higher among patients with AIDS in Africa and South Asia than in United States, whereas it appears less frequently in Europe (Levitz SM et al 1991).

In the developed world, the introduction of potent antiretroviral therapies resulted in a decrease in the incidence of opportunistic infections associated with AIDS. In 1994, the annual prevalence of cryptococcosis was calculated between $6.1 \%$ and $8.5 \%$ among HIV infected individuals in New York City (Currie Bp et al 1994).

Considered an AIDS-defining condition (AbadiJ,DelValle L et al 2006), cryptococcal meningitis is the most common fungal infection of the central nervous system(CNS) and the third most frequent neurological complication in AIDs patients. The ecological agent is the basidiomycetes yeast-Cryptococcus neoformans, which can also infect, although with significantly lower incidence, people with decreased immunity such as individuals with sarcoidosis, lympho-proliferative disorders, and those undergoing immunosuppressive therapies (Korfel, A et al 1998).Currently the incidence of AIDS patients developing cryptococcosis is relatively low in 
western countries, but it is almost uniformly fatal and can cause up to $30 \%$ mortality in AIDS patients in regions where Highly Active Antiretroviral Treatment (ART) is not available such as South East Asia and East Africa(Helbok R, Holmes CB et al 2003).

\section{2: The route of infection and organs involved}

When Cryptococcus neoformanswas discovered more than a century ago, this encapsulated yeast was isolated independently from peach juice(Sanfelice F.et al 1894), and from the tibial lesion of a patient(Buschke A. et al 1895), indicating that this fungus is not an obligate human pathogen. Cryptococcosis occurs in both animals and humans, but animal-to-human or human-to-human transmission has not been documented, other than rare examples of iatrogenic transmission. The major environmental sources of $\mathrm{C}$. neoformans are eucalyptus trees and decaying wood, (Callejas A et al1998) or either soil contaminated with pigeon guano (the excrement of sea birds). Human infection is thought to be acquired by inhalation of airborne propagules from an environmental source (Swinne, D et al 1991). Cryptococcus neoformans can colonize the host respiratory tract without producing any disease infection; it is typically asymptomatic, and can be either cleared or enter a dormant latent form. When host immunity is compromised, the dormant form can be reactivated and disseminate haematogenously to cause systemic infection. Cryptococcusneoformans can infect or spread to any organ to cause localized infections involving the skin, eyes, myocardium, bones, joints, lungs, prostate gland, or urinary tract (Ghigliotti, G et al 1995), in addition to its tendency to infect the central nervous system. Persistent cryptococcal infection is likely due to relapse rather than re-infection(Brandt ME, et al 1996).

A survey of children 2 years and older in New York City showed that most of them had serological evidence of asymptomatic C. neoformans infection (Goldman DL et al 2001). Serial isolates of C.neoformans from AIDS patients exhibit no change in genotype, indicating that persistent cryptococcal infection is caused by relapse rather than re-infection (Igreja RP et al 2004). Evidence for dormant C. neoformans infection is derived from a study of patients in France who acquired the fungus originally in Africa, where they lived approximately 10 years prior to moving to Europe. All the isolates from these patients had Randomly Amplified Polymorphic DNA (RAPD) patterns different from those of isolates from European, American, or Asian patients (GarciaHermoso, D et al 1999).

In a study in Brazil, C. neoformans cells were serially isolated from AIDS patients over periods ranging from 18 to 461 days to determine whether the original strain persisted or if re-infection with a new strain had occurred. Isolates were collected from different body sites and also from blood and cerebrospinal fluid from each patient to determine if infection by different strains occurred simultaneously. Except in two patients, all isolates obtained from the same patient exhibited identical PCR profile independent of times of isolation or body site and each patient carried unique genotypes of $\mathrm{C}$.neoformans strain in most cases but they were infected with more than one strain in rare cases (Brandt ME et al 1996). This conclusion is also supported by other similar studies showing that genotypes of $\mathrm{C}$. neoformans strains from the same patient at different times and from different body sites exhibited identical patterns (Sorrel TC et al 1996).

\section{3: Epidemiology}

Although more men are reported to develop cryptococcal disease, the male- to -female ratio essentially is 1:1, when adjusted for the male predominance in HIV infection. Cryptococcusneoformans in children with AIDS is less common, with prevalence of approximately $1.4 \%$ (Abadi,J et al 1999).

More than three fourths of the cases associated with AIDS develop when the CD4 T-lymphocyte count falls below 50 cells / $\mu$ l.(Pinner RW et al 1995). In 1996 retrospective review of 65 AIDS patients with cryptococcal meningitis in France showed that the median CD4 cells counts were 46 cells/ $\mu 1$. Cryptococcusneoformans was the initial AIDS defining illness in 63\% (Darras -Joly C.et al 1996).

\subsection{1: Factors Leading to the Disease}

Factors independently associated with the disease included being in a warehouse within the previous month and having sexual contact with an infected drug user. Other factors were cigarette smoking and immunosuppression as seen in the late stage of AIDS or in WHO stage four patients (Oursler KA, et al 1999).

\section{4: Laboratory Diagnosis}

Diagnosis is confirmed by isolation of Cryptococcus from a sterile body site, by histopathologic analysis, or by detection of cryptococcal capsular antigen. Cryptococcus Antigen in serum is usually indicative of systematic disease and correlates with fungal burden. Detection of Cryptococcus antigen in either serum or CSF has $>95 \%$ sensitivity and specificity in the diagnosis of true invasive cryptococcal infection (Currie Bp et al 1993). False positive results can occur secondary to infection with Trichosporonbeigelii, which cross-react with the antigen. In addition, false positive results have occurred secondary to residual disinfectant on laboratory test slide and inactivated pronase in a test kit (Blevin, Lb et al 1995). 


\section{5: Treatment}

Based on the results of the Mycoses Study Group (MSG) and AIDS Clinical Trials Group (ACTG), clinical trials with amphotericin B $(0.7 \mathrm{mg} / \mathrm{kg}$ intravenously per day) plus flucytosine $(100 \mathrm{mg} / \mathrm{kg} / \mathrm{day})$ for 2 weeks, followed by fluconazole (400mg daily) for 8 weeks of consolidated therapy and 200mg daily for maintenance therapy, is recommended as first-line therapy for AIDS patients with cryptococcal meningitis.

\section{1: Ethical Consideration}

\section{Protocol Followed}

The study got clearance fromMedical superintendent Kapkatet District Hospital,School of Health Sciences Mt Kenya University, and in-charges of both CCC and Medical Laboratory Services of Kapkatet District Hospital.

\section{2: Selection Criteria}

\subsection{1: Inclusion criteria}

All HIV patients of all ages who attended CCC both males and females at Kapkatet District Hospital during the study period were included in the sample.

\subsection{2: Exclusion criteria}

i. All other patients with other conditionsattending special clinics.

ii. HIV patients not attending the CCC.

\section{1: Study Area}

\section{Methodology}

The study was conducted within the Laboratory Department of Kapkatet District Hospital which serves residents of Bureti, Konoin, Sotik, Borabuand Trans Mara districts with an estimated clientele of approximately 300,000 people.

\section{2: Study Design}

This was a descriptive cross-sectional study involving collection and analysis of blood samples.

\section{3: Study Population}

The targeted population was selected HIV patients attending Comprehensive Care Clinic at Kapkatet District Hospital during the study period.

\section{4: Sampling Techniques \\ 5.4.1: Determination of Sample Size}

The sample size was determined by using purposive sampling method where $10 \%$ of the patients enrolledin CCC register (3000 of whom were in the register) were included in the sample starting from all the willing HIV patients who reported on the date the study commenced and continued until the $300^{\text {th }}$ patient was enrolled.

\section{5: Data Collection Tool}

Observational checklist was used for data collection.

\section{6: Data Collection Procedure}

Blood samples which are normally collected every month from HIV/AIDS clients for routine investigations for CD4,Chemistry and Haematology were used to determine whether there was the presence of Cryptococcus neoformans. Results were then posted to individual observational checklists.

\section{7: Data Analysis and Presentation}

Data was collected manually, analysed using excel and presented in form of tables, bar graphs, pie charts and graphs.

\section{Results}

The number of enrolled respondents were 300 and the results were as follows: 6.1: The proportion of patients with cryptococcal infection among HIV/AIDS patients enrolled in CCC register who came for review at Kapkatet District Hospital 
The response in respect to the proportion of patients with cryptococcal infection among HIV/AIDS patients enrolled in CCC register who came for review at Kapkatet District Hospital was as provided in Table 6.1 which shows the Crag Results.

Table 6.1: Crag Results -Proportion of Patients with Cryptococcosis

\begin{tabular}{|l|c|c|}
\hline Response & Frequency & Percentage \\
\hline Negative & 296 & 99 \\
\hline Positive & 4 & 1 \\
\hline Total & 300 & 100 \\
\hline
\end{tabular}

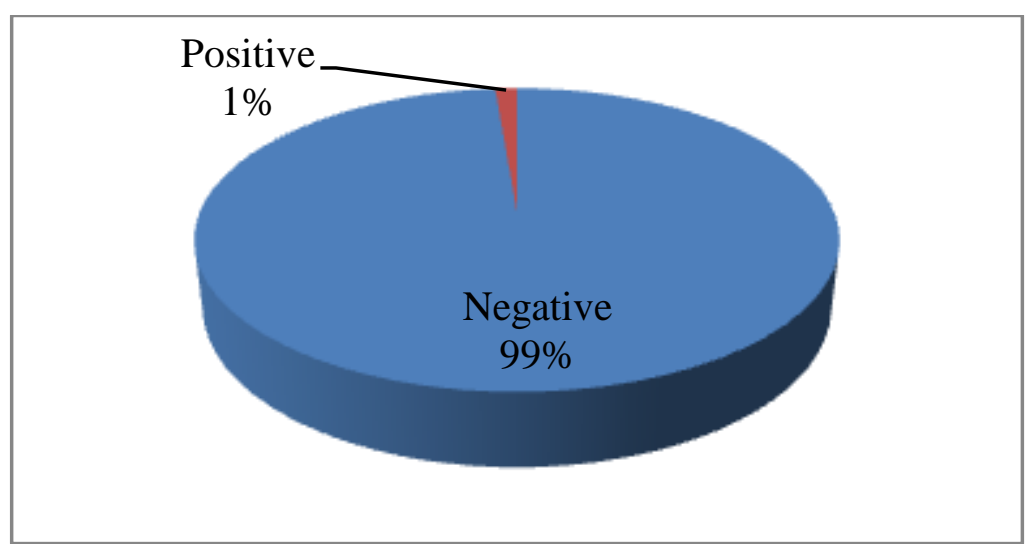

Figure 6.1:Proportion of Patients with Cryptococcosis

The findings show that $99 \%$ of the 300 HIV/AIDS patients enrolled in CCC register who came for review at Kapkatet District Hospital had negative Crag results, while only $1 \%$ had positive results.

6.2: The proportion of patients with HIV infection according to age

The response in respect to the patient's age was as given in Table 6.2 and Figure 6.2 below.

Table 6.2: Age of Patients

\begin{tabular}{|l|c|c|}
\hline Response & Frequency & Percentage \\
\hline $1-10$ years & 14 & 4.7 \\
\hline $11-20$ years & 39 & 13 \\
\hline $21-30$ years & 60 & 20 \\
\hline $31-40$ years & 95 & 31.7 \\
\hline $41-50$ years & 64 & 21.3 \\
\hline $51-60$ years & 21 & 7 \\
\hline $61-70$ years & 7 & 2.3 \\
\hline Total & 300 & 100 \\
\hline
\end{tabular}

The findings show that: $31.7 \%$ of the respondents were aged between the age of 31 and 40 years, $21.3 \%$ were aged between 41 and 50 years, $20 \%$ were aged between 21 and 30 years, $13 \%$ were aged between 11 and 20 years, $7 \%$ were aged between 51 and 60 years, $4.7 \%$ were aged between 1 and 10 years, while $2.3 \%$ were aged between 61 and 70 years.

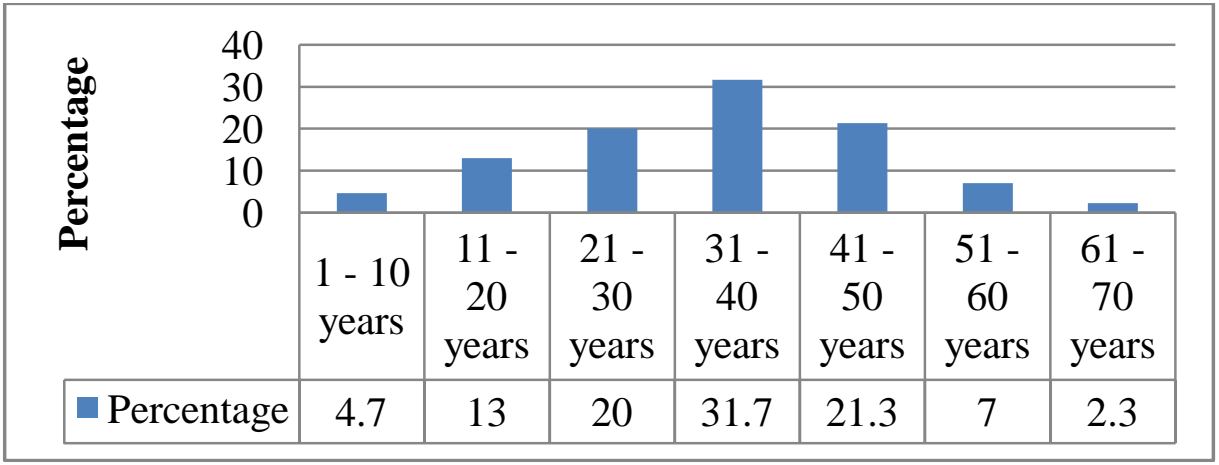

Figure 6.2: Age of Patients 
Detection Of Cryptococcus Neoformans Amongst HIV Patients Attending Comprehensive Care ...

As shown in the figure above HIV AIDS cases at Kapkatet District Hospital are concentrated between 21 and 50 years $(73 \%)$, with the highest proportion concentrated between 31 and 40 years.

\section{3: Cross tabulation showing the relationship between CD4 Intervals and Gender}

A crosstab showing the relationship between CD4 intervals and gender was as given in Table6.3.

Table 6.3: Cross tabulation showing the relationship between CD4 Intervals and Gender

\begin{tabular}{|c|c|c|c|c|}
\hline & \multirow{3}{*}{ Below 100} & \multicolumn{2}{|c|}{ Sex } & \multirow[t]{2}{*}{ Total } \\
\hline & & Male & Female & \\
\hline \multirow[t]{10}{*}{ CD4 Intervals } & & 12 & 5 & 17 \\
\hline & $101-200$ & 19 & 12 & 31 \\
\hline & $201-300$ & 22 & 27 & 49 \\
\hline & $301-400$ & 28 & 31 & 59 \\
\hline & $401-500$ & 20 & 16 & 36 \\
\hline & $501-600$ & 15 & 19 & 34 \\
\hline & $601-700$ & 11 & 8 & 19 \\
\hline & $701-800$ & 13 & 18 & 31 \\
\hline & $901-100$ & 4 & 4 & 8 \\
\hline & Above 1000 & 7 & 9 & 16 \\
\hline \multicolumn{2}{|l|}{ Total } & 151 & 149 & 300 \\
\hline
\end{tabular}

The findings show that the study population was composed 151 males(51\%) and 149 females (49\%) as illustrated in Table 6.3 above and Figure 6.3.1 below.

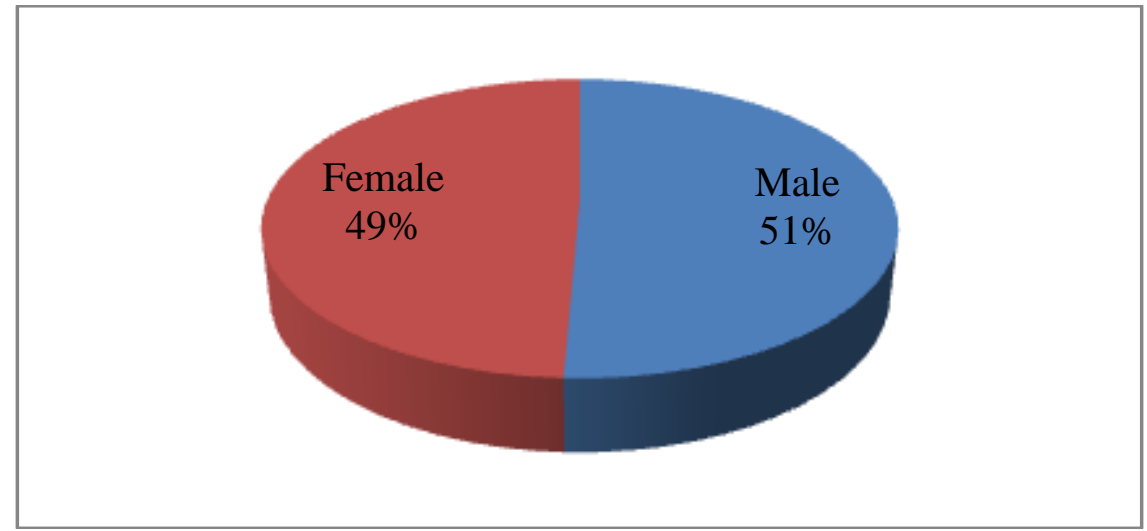

Figure 6.3.1: Gender of the HIV patients used in the study

Figure 6.3.1 shows that there were slightly more males used in the study compared to the female counterparts, however the difference was very minimal to influence the results.

The findings of Table 6.4also show that the highest number of HIV patients (59 out 300) had a CD4 count between of 301 and 400; out which 31 were female and 28 were male. This was followed by 49 out 300 HIV patientsstudiedwho had a CD4 count of between 201 and 300.36 out of 300 had a CD4 count of between 401 and 500 and 31 out of 300 had a CD4 count of between 101 and 200. The findings also show that 203 out of 300 patients $(68 \%)$ had a CD4 count of 301 or above. The interpretation was that 97 out of $300(32 \%)$ of the HIV/AIDS patients enrolled in CCC register who came for review at Kapkatet District Hospital had a CD4 count of 300 or below. This is further presented graphically in Figure 6.4 below.

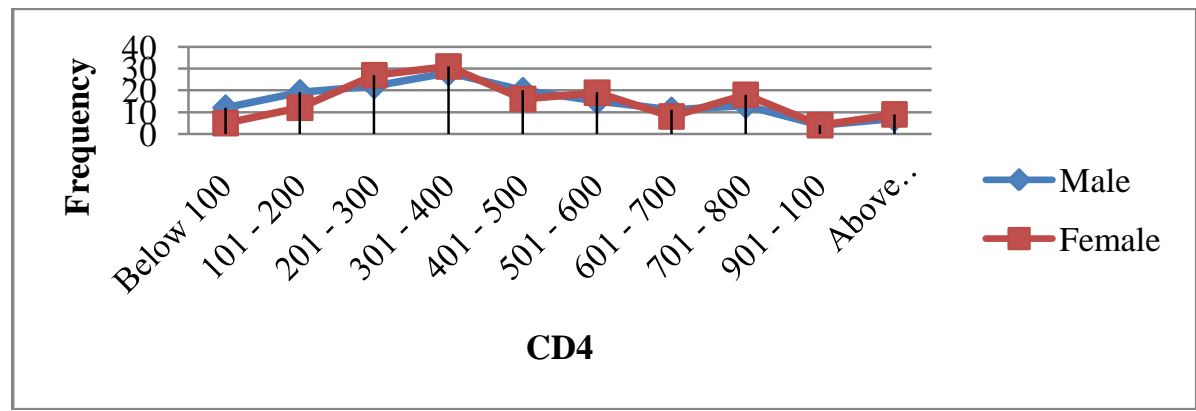

Figure 6.3.2: Relationship between CD4 Intervals and Gender 
6.4: Proportion of patients with cryptococcal infection according to age

The response in respect to the proportion of patients with cryptococcal infection according to age was as given in Table 6.4 and Figure 6.4 below.

Age (Yrs.) * Crag Results Cross tabulation

Table 6.5: Proportion of patients with cryptococcal infection according to age

\begin{tabular}{|c|c|c|c|c|}
\hline & \multicolumn{2}{|c|}{ Crag Results } & \multirow[t]{2}{*}{ Total } \\
\hline & & Negative & Positive & \\
\hline \multirow[t]{7}{*}{ Age (Yrs.) } & $1-10$ years & 14 & 0 & 14 \\
\hline & $11-20$ years & 39 & 0 & 39 \\
\hline & $21-30$ years & 59 & 1 & 60 \\
\hline & $31-40$ years & 94 & 1 & 95 \\
\hline & $41-50$ years & 63 & 1 & 64 \\
\hline & $51-60$ years & 20 & 1 & 21 \\
\hline & $61-70$ years & 7 & 0 & 7 \\
\hline \multicolumn{2}{|l|}{ Total } & 296 & 4 & 300 \\
\hline
\end{tabular}

The findings show that 4 out of $300(1 \%)$ HIV patients had positive Crag results and distributed as follows:

1 - in age group between 21 and 30 years,

1- inage group between 31 and 40 years,

1 - inage groupbetween 41 and 50 years and

1 - inage group between 51 and 60 years.

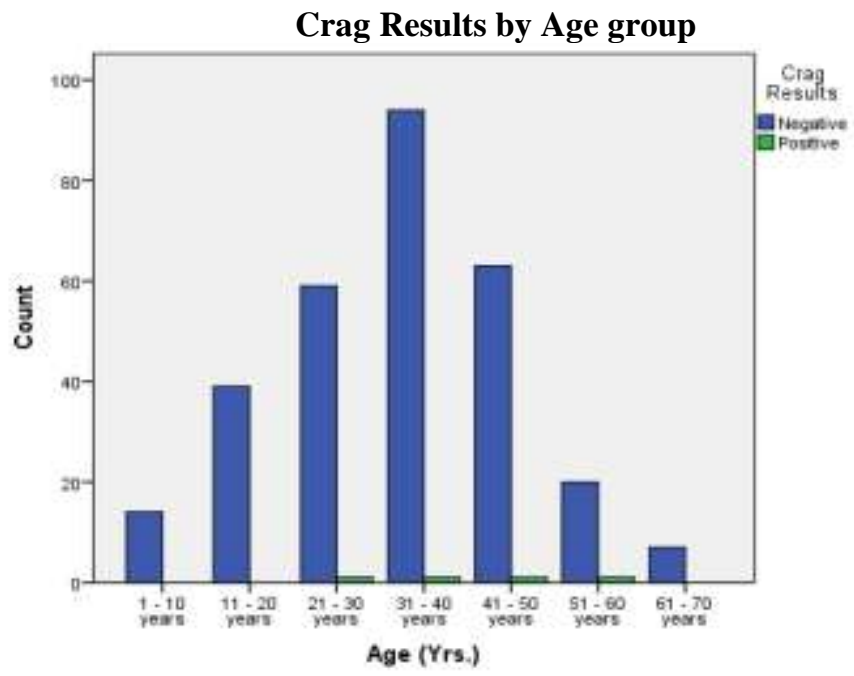

Figure 6.3:Patients with cryptococcal infection according to age

6.5:Proportion of patients with cryptococcal infection according to gender

The response in respect to the proportion of patients with cryptococcal infection according to genderwas as given in Table 6.5 .

Table 6.5: Proportion of patients with cryptococcal infection according to gender

\begin{tabular}{|c|c|c|c|c|c|}
\hline & \multicolumn{3}{|c|}{ Crag Results } & \multirow[t]{2}{*}{ Total } \\
\hline & & Negative & Positive & Percentage positive & \\
\hline \multirow[t]{2}{*}{ Sex } & Male & 150 & 1 & 25 & 151 \\
\hline \multirow{2}{*}{\multicolumn{2}{|c|}{ Total }} & 146 & 3 & 75 & 149 \\
\hline & & 296 & 4 & 100 & 300 \\
\hline
\end{tabular}

The findings show that $75 \%$ of HIV patients that were positive were female, while $25 \%$ weremale.

\section{6: Characteristics of HIV patients that showed positive Crag results}

The response in respect to the characteristics of HIV patients that showed positive Crag results was as given in Table 6.6. 
Detection Of Cryptococcus Neoformans Amongst HIV Patients Attending Comprehensive Care ...

Table 6.6: Characteristics of HIV patients that showed positive Crag results

\begin{tabular}{|c|c|c|c|}
\hline Age (Yrs.) & Sex & CD4 & $\begin{array}{c}\text { Percentage proportion out of } \\
\text { Positive Crag results }\end{array}$ \\
\hline $\mathbf{3 5}$ & Male & 531 & $25 \%$ \\
\hline $\mathbf{2 2}$ & Female & 1609 & $25 \%$ \\
\hline $\mathbf{4 6}$ & Female & 214 & $25 \%$ \\
\hline $\mathbf{5 4}$ & Female & 905 & $25 \%$ \\
\hline & & 814.75 & $100 \%$ \\
\hline
\end{tabular}

The findings show that 1 out of 4 Crag positive patients had a CD4 count of less than 300 CD4+ (214) cells/micro litres of blood while 3 out of the 4 patients had CD4 count of more than 300 CD4+. The Crag positive patient with the highest CD4+ cells/micro litres of blood was female aged 22 years (CD4+ of 1609 cells/micro litres of blood), while the patient who had CD4 count of less than 300 had a count of 214CD4+ cells/micro litres of blood, was a female aged 46 years.

6.7: A Pearson Product Moment Correlation showing the relationship between Crag Results and CD4 A Pearson Product Moment Correlation showing the relationship between Crag Results and CD4 was as given in Table6.8.

Table 6.7: Pearson Product Moment Correlation showing the relationship between Crag Results and CD4

\begin{tabular}{|c|c|c|c|}
\hline & & Crag Results & CD4 \\
\hline \multirow[t]{3}{*}{ Crag Results } & Pearson Correlation & 1 & $.134 *$ \\
\hline & Sig. (2-tailed) & & .020 \\
\hline & $\mathrm{N}$ & 300 & 300 \\
\hline \multirow[t]{3}{*}{ CD4 } & Pearson Correlation & $.134 *$ & 1 \\
\hline & Sig. (2-tailed) & .020 & \\
\hline & $\mathrm{N}$ & 300 & 300 \\
\hline
\end{tabular}

The results show that there is a positive Pearson Correlation between Crag Results and CD4 at 0.134. The correlation coefficient is a measure of correlation strength and can range from -1.00 to +1.00 (Cherry, 2012). Positive Correlations appear when variables increase or decrease at the same time. A correlation coefficient close to +1.00 indicates a strong positive correlation. The correlation of 0.134 though positive is not a strong positive. This implies that there was a weak relationship between Crag Results and CD4.

The hypothesis that it is generally accepted that most patients who are immuno-suppressed and have HIVtend to have Cryptococcus neoformans infectionis not correctand therefore the research hypothesis is rejected, given that only 4 out $300(1 \%)$ patients who were immuno-suppressed and had HIV were found to have Cryptococcus neoformans infection.

\section{Conclusions}

1. It was established that of the patients who came to Kapkatet District hospital $1 \%$ had cryptococcal infection.

2. The proportion of patients with cryptococcal infection according to age and gender showed that females were more affected compared to males and their ages ranged between 21 and 60 years.

3. Of the 97 patients with less than $300 \mathrm{CD} 4+$ cells/micro liters of blood only 1 had cryptococcal infection.

4. The hypothesis that it is generally accepted that most patients who are immuno-suppressed and have HIVtend to have Cryptococcus neoformans infectionis not correct.

\section{Recommendations}

1. Crag test should be done as a baseline investigation for all HIV clients irrespective of their CD4+ levels without necessarily stressing on those with CD4+ levels above 300 cells/micro liters of blood.

2. More research to be done to include more patients and other parameters like vegetation and the environment.

[1]. UNAIDS/WHO. AIDS Epidemic Update. Geneva: WHO, 2006

[2]. Bicanic T, Harrison TS. Cryptococcal meningitis. Br Med Bull 2005; 72: 99-118.

[3]. Robinson PA, Bauer M, Leal MA, Early mycology treatment failure in AIDS associated cryptococcal meningitis. ClinInfect Dis 2000;30: 710-718

[4]. Mwaba P, Mwansa J, Chintu C. Clinical presentation, natural history and cumulative death rates of 230 adults with primary cryptococcal meningitis in Zambian AIDS patients treated under local conditions. Postgrad Med J 2001; 77: 769-773 
Detection Of Cryptococcus Neoformans Amongst HIV Patients Attending Comprehensive Care ...

[5]. Moosa MY, Coovadia YM. Cryptococcal meningitis in Durban, South Africa: a comparison of clinical features, laboratory findings and outcomes for Human Immunodeficiency Virus (HIV)-positive and HIV negative patients. Clin Infect Dis 1997; $24: 131-134$.

[6]. Iyer RS, Banker DD. Cryptococcal meningitis in AIDS. Indian J Med Sci 2002; 56: 593-597.

[7]. Schaars CF, Meintjes GA, Morroni C, Post FA, Maartens G. Outcomes of AIDS-associated cryptococcal meningitis initially treated with $200 \mathrm{mg} /$ day or $400 \mathrm{mg} /$ day of fluconazole. BMC Infect Dis 2006; 6:118.

[8]. Aberg JA, Powderly WG. Cryptococcosis. AdvPharmacol 1997; 37:215-251.

[9]. Levltz SM. The ecology of Cryptococcus neoformans and the epidemiology of cryptococcosis. Rev Infect Dis 1991; 13:1163-1169.

[10]. Speed B, Dunt D. Clinical and host differences between infections with the two varieties of Cryptococcus neoformans. ClinInfect Dis $1995 ; 21: 28-34$.

[11]. Dismukes WE. Cryptococcal meningitis in AIDS. J Infect Dis 1988; 57:624 628.

[12]. Ellis DH, Pfeiffer TJ. Ecology, life cycle and infectious propagule of Cryptococcusneoformans. Lancet 1990; 336:923-925.

[13]. Chemiak R, Morris LC, Belay T, Spitzer ED, Lasadevall A. Variation in the structure of glucuronoxylomannan in isolates from patients with recurrent cryptococcal meningitis. Infect Immun 1995; 63: 1899-1905.

[14]. King JW, Markanday A, Khan A. Cryptococcosis. eMedicine. Last updated 26 May 2005. http://www.eMedicine.com.

[15]. Mitchell TG, Perfect JR. Cryptococcosis in the era of AIDS - 100 years after discovery of Cryptococcus neoformans. Clin MicrobiolRev 1995; 8: 515-548.

[16]. McGuire D, Bromley E, Aberg J, et al. Focal posterior-hemisphere invasive cryptococcal encephalitis - a distinct clinicalneuroimaging entity complicating cryptococcal meningitis in AIDS. Program and Abstracts of the 122ndAnnual Meeting of the American NeurologicalAssociation. September 28th -October 1st, 1997. San Diego, California.

[17]. Currie BP, Freundlich LF, Soto MA, Casadevall A. False negative cerebrospinal fluid cryptococcal latex agglutination testswith culture positive cryptococcal meningitis. J ClinMicrobiol 1993; 31: 2519-2522.

[18]. Tanner DC, Weinstein MP, Fedorciw B, Joho KL, Thorpe JJ, Reller L. Comparison of commercial kits for detection of cryptococcal antigen. J ClinMicrobiol 1994;32:1680-1684.

[19]. Grant AD, De Cock KM. ABC of AIDS: HIV infection and AIDS in the developing world. BMJ 2001;322:1475-1478.

[20]. Ennis DM, Saag MS. Cryptococcal meningitis in AIDS. Hospital Practice 1993; 28: 99-112.

[21]. Rozenbaum R, Gonclaves AJ. Clinical epidemiology study of 171 cases of cryptococcosis. Clin Dis 1994; 18: 369-380 LevitzSM 1991).

[22]. Pinner RW, Haijeh RA, Powderly WG. Prospects for preventing crytococcosis in persons infected with human immunodeficiency virus. Clin infect Dis $1995 ; 21$ Suppl $1:$ S103-7.

[23]. Darras-Joly C, Chevret S, Wolff M, Matheron S, longuet P, casalino E, joly V. chochillon C, Bedos JP. Cryptococcus neoformans infection in France : epidemiologic features of and early prognostic parameters for 76 patients who were infected with human immunodeficiency virus. Clin Infect Dis $1996 ; 23: 369-76$. 